\title{
Spineurs, Opérateurs de Dirac et Variations de Métriques *
}

\author{
Jean-Pierre Bourguignon ${ }^{1}$ and Paul Gauduchon ${ }^{2}$ \\ ${ }^{1}$ Centre de Mathématiques, Ecole Polytechnique, U.R.A. 169 du C.N.R.S., F-91128 Palaiseau \\ Cedex, France \\ ${ }^{2}$ Département de Mécanique, Université Paris VI, U.R.A. 766 du C.N.R.S., F-75252 Paris \\ Cedex 05, France
}

Received June 21, 1991

\begin{abstract}
In this article a geometric process to compare spinors for different metrics is constructed. It makes possible the extension to spinor fields of a variant of the Lie derivative (called the metric Lie derivative), giving a geometric approach to a construction originally due to Yvette Kosmann. The comparison of spinor fields for two different Riemannian metrics makes the study of the variation of Dirac operators feasible. For this it is crucial to take into account the fact that the bundle in which the sections acted upon by the Dirac operators take their values is changing. We also give the formulas for the change in the eigenvalues of the Dirac operator. We conclude by giving a few cases in which an eigenvalue is stationary.
\end{abstract}

\section{Introduction}

Le problème que nous traitons dans cet article est celui de la dépendance des spineurs et de l'opérateur de Dirac par rapport à la métrique. Malgré son importance tant en Physique (lorsque la Gravité, donc la métrique sur l'espace-temps, est couplée à d'autres interactions) qu'en Mathématiques (lorsqu'on veut utiliser l'opérateur de Dirac comme outil d'investigation en Géométrie Riemannienne à partir de la formule fondamentale de Lichnerowicz, cf. [16]), cette question est laissée le plus souvent de côté dans la littérature.

Cet état de fait peut éventuellement être attribué à une lecture restrictive du théorème par lequel Elie Cartan termine le chapitre "Champs de spineurs en Géométrie riemannienne" de [6] (et le livre lui-même) et qu'il reprend dans une note terminant la version publiée de sa Notice sur les travaux scientifiques [7]: "Les difficultés (rencontrées quand on a voulu étendre les équations de Dirac de la Relativité Restreinte à la Relativité Générale) sont insurmontables si l'on veut

* Recherche soutenue par le programme européen C.E.E. G.A.D.G.E.T. SC1-0105-C 
conserver la technique classique de la Géométrie Riemannienne: il est impossible, un système de coordonnées étant donné dans l'espace-temps, de représenter... un champ de spineurs par un nombre fini de composantes..." (cf. [18] pour un examen de certaines des conséquences physiques). Les succès de l'utilisation de l'opérateur de Dirac comme opérateur elliptique-modèle dans les nouvelles approches du Théorème de l'Indice, où la référence à la métrique passe au second plan, peuvent en être une autre explication. Le problème de cette dépendance a été traité dans un article d'E. Binz et R. Pferschy (cf. [5] et aussi [20]). Nous complétons leurs résultats et donnons une solution géométrique de ce problème.

Dans le cadre algébrique, la notion de spineur est définie seulement après qu'une métrique ait été choisie, et la difficulté de comparer les spineurs attachés à deux métriques différentes est reliée au fait algébrique suivant (qui est élémentaire mais peu souvent mentionné): les représentations de dimension finie du groupe spinoriel (revêtement à deux feuillets du groupe spécial orthogonal) que sont les représentations spinorielles ne sont pas des représentations du revêtement à deux feuillets du groupe linéaire car toutes les représentations de dimension finie de ce groupe sont déjà des représentations du groupe linéaire lui-même (cf. [21]).

$\mathrm{Si}$, dans le cadre algébrique, définir des objets géométriques à un isomorphisme près est sans conséquences, il n'en va plus de même lorsqu'on considère la théorie "à paramètres", i.e. lorsqu'on regarde des sections de fibrés de tels objets et que l'on veut les différentier.

C'est précisément le cas pour les champs de spineurs sur lesquels est défini naturellement l'opérateur de Dirac. Le formalisme que nous développons pour analyser cette dépendance a l'avantage d'être géométrique, et de permettre l'évaluation des variations de l'opérateur de Dirac, puis ultérieurement de la dépendance de ses valeurs propres par rapport à la métrique.

L'article est organisé comme suit: dans la Sect. I, nous établissons l'équivalence naturelle entre structures euclidiennes sur un espace vectoriel sur laquelle repose tout l'article. Cette construction permet de définir une notion de dérivation de Lie métrique à laquelle nous consacrons la Sect. II, mais surtout elle rend possible la comparaison entre spineurs pour différentes métriques ce qui permet d'étendre la notion de dérivée de Lie métrique à ces champs. Une telle extension avait déjà été introduite par Yvette Kosmann dans [14]. Nous donnons ensuite dans la Sect. III les formules explicites décrivant la variation de l'opérateur de Dirac et de ses valeurs propres.

Le lecteur peu familier avec les notions de spineur et d'opérateur de Dirac pourra se référer utilement à [12] et au récent ouvrage [15].

Les auteurs tiennent à remercier Michael F. Atiyah, Oussama Hijazi, André Lichnerowicz et Raymond Stora pour des discussions en relation avec le sujet de cet article. Le premier auteur a profité de l'hospitalité du département de Mathématiques d'Ohio State University lors de la rédaction de la première version de ce manuscrit.

\section{Equivalence Naturelle Entre Structures Euclidiennes sur un Espace Vectoriel}

Sur un espace vectoriel $V$ de dimension $n$, il est bien connu que deux métriques euclidiennes $g$ et $h$ (nous notons $\mathscr{M} V$ le cône de ces métriques) sont l'image l'une de l'autre par une application linéaire $l$ (i.e. nous avons $h=l^{*} g$ ). 
Le problème qui nous préoccupe est de rendre la définition de $l$ canonique, ce qui est indispensable dès qu'on considère une théorie "à paramètres", i.e. des métriques riemanniennes définies sur une variété. Pour l'usage géométrique ultérieur, il est utile de formuler cette correspondance de la façon suivante.

Nous notons $\mathscr{B} V$ l'espace des bases linéaires de $V$ que nous considérons, dans l'esprit de la théorie des fibrés principaux, comme l'ouvert des éléments inversibles de l'espace $L\left(\mathbb{R}^{n}, V\right)$ des applications linéaires de $\mathbb{R}^{n}$ dans $V$ en identifiant un élément $f$ de cet ouvert avec la base $\left(f\left(e_{i}\right)\right)$ (où $\left(e_{i}\right)$ est la base canonique de $\mathbb{R}^{n}$ ). Le groupe $\mathbb{R} \mathrm{Gl}_{n}$ des automorphismes de $\mathbb{R}^{n}$ opère transitivement à droite sans point fixe sur $\mathscr{B} V$ de telle sorte que $\mathscr{B} V$ peut s'identifier à $\mathbb{R} \mathrm{Gl}_{n}$ dans lequel on a "perdu" l'identité. (Le groupe $G l(V)$ des automorphismes de $V$ opère lui aussi sur $\mathscr{B} V$ mais transitivement à gauche.) Par ailleurs, si $g$ est une métrique euclidienne sur $V$, nous notons $\mathscr{B}_{g} V$ l'espace des bases $g$-orthonormées, sur lequel agit transitivement à droite et sans point fixe le groupe orthogonal $\mathrm{O}_{n}$ ce qui fait de $\mathscr{B}_{g} V$ une sous-variété de $\mathscr{B} V$ (et le groupe orthogonal de $g$, noté $O_{g} V$, opère lui à gauche). On peut remarquer que, si $f \in \mathscr{B}_{g} V$, et si $e$ désigne la métrique euclidienne standard de $\mathbb{R}^{n}$, alors

$$
g=\left(f^{-1}\right)^{*} e .
$$

Pour tout couple de métriques $g$ et $h$, nous noterons génériquement $H_{g}=g^{-1} \cdot h$ l'automorphisme $g$-symétrique de $V$ associé à $h$ par la dualité définie par $g$, i.e. défini, pour tout $u, v \in V$, par

$$
h(u, v)=g\left(H_{g}(u), v\right)
$$

Nous avons alors

Proposition 1 (cf. [5]). Soient $g$ et $h$ deux métriques euclidiennes sur un espace vectoriel $V$. L'application $b_{h}^{g}$ définie sur la base g-orthonormée $f$ par $b_{h}^{g}(f)=H_{g}^{-1 / 2} \circ f$ (où, pour l'endomorphisme symétrique positif $A, A^{1 / 2}$ désigne sa racine carrée positive) est un difféomorphisme de $\mathscr{B}_{g} V$ sur $\mathscr{B}_{h} V$ qui est une transformation naturelle au sens suivant:

i) $b_{h}^{g}=\left(b_{g}^{h}\right)^{-1}$;

ii) $b_{g}^{h}$ commute à l'action à droite de $\mathrm{O}_{n}$ sur $\mathscr{B} V$;

iii) pour toute courbe différentiable de métriques $t \mapsto g_{t}$ sur $V, b_{g_{t}}^{g}$ est une isotopie de $\mathscr{B}_{g} V$ sur $\mathscr{B}_{g_{t}} V$.

Preuve. Vérifions d'abord les propriétés algébriques. Si $f$ est $g$-orthonormée, $g=\left(f^{-1}\right)^{*} e$, d'où

$$
\left(\left(b_{h}^{g}(f)\right)^{-1}\right)^{*} e=\left(H_{g}^{1 / 2}\right)^{*}\left(f^{-1}\right) * e=\left(H_{g}^{1 / 2}\right)^{*} g=h,
$$

puisque, pour $v \in V$,

$$
\left(\left(H_{g}^{1 / 2}\right)^{*} g\right)(v, v)=g\left(H_{g}^{1 / 2}(v), H_{g}^{1 / 2}(v)\right)=h(v, v) .
$$

On a bien $b_{h}^{g}=\left(b_{g}^{h}\right)^{-1}$ puisque $G_{h}=h^{-1} \cdot g$ agissant sur $v \in V$ a pour $h$-produit scalaire avec $w \in V$,

$$
h\left(G_{h}(v), w\right)=g(v, w)=g\left(H_{g} \circ\left(H_{g}\right)^{-1}(v), w\right)=h\left(H_{g}^{-1}(v), w\right) .
$$

Pour ii), si $U \in \mathrm{O}_{n}$, nous avons

$$
b_{h}^{g}(f \circ U)=H_{g}^{-1 / 2} \circ f \circ U=\left(H_{g}^{-1 / 2} \circ f\right) \circ U=b_{h}^{g}(f) \circ U .
$$


La fin de la preuve est alors une conséquence facile du fait que l'application $S \mapsto S^{-1 / 2}$ est un difféomorphisme du cône des transformations symétriques positives sur lui-même.

Il suffit enfin de vérifier que, $g$ étant fixée, l'application $h \mapsto H_{g}$ est différentiable, ce qui est évident.

Remarques. i) En général, si $l_{1}$ et $l_{2}$ sont deux isomorphismes linéaires de $V$, nous avons

$$
b_{\left(l_{2} l_{1}\right)^{*} g}^{g} \neq b_{\left(l_{2} \circ l_{1}\right)^{*} g}^{l_{*}^{*} g} b_{l_{2}^{*} g}^{g},
$$

car, si nous posons $h=l_{2}^{*} g, k=\left(l_{2} \circ l_{1}\right)^{*} g, K_{g}^{-1 / 2} \neq K_{h}^{-1 / 2} \circ H_{g}^{-1 / 2}$ sauf à ce que soient vérifiées des propriétés de commutation sur lesquelles nous revenons ultérieurement.

ii) L'expression de $b_{h}^{g}$ se réduit à $b_{a g}^{g}=a^{-1}$ Id lorsque $h=a^{2} g$ avec $a \in \mathbb{R}^{+}$.

Le contenu géométrique de l'application $b_{h}^{g}$, peu clair dans la formulation de la Proposition 1, est mis en lumière par la Proposition 2 qui en constitue une définition alternative. Le développement qui suit est une variation sur le théorème de décomposition polaire selon lequel $\mathbb{R ~ G l}_{n}$ est difféomorphe au produit de $\mathrm{O}_{n}$ par le cône $\mathscr{C}_{n}$ des matrices positives par l'application qui décompose toute matrice $A$ en produit d'une matrice orthogonale $U$ et d'une matrice symétrique positive $S\left(=\left({ }^{t} A A\right)^{1 / 2}\right)$.

Considérons la projection $p: \mathscr{B} V \rightarrow \mathscr{M} V$ définie par $p(f)=\left(f^{-1}\right)^{*} e$. Le théorème de décomposition polaire implique en particulier que cette fibration est triviale. Sa fibre en $g$ est précisément $\mathscr{B}_{g} V$. L'espace tangent $T_{f} \mathscr{B} V$ s'identifie à $L\left(\mathbb{R}^{n}, V\right)$. Soient $\mathscr{A}_{f} V$, respectivement $\mathscr{S}_{f} V$, le sous-espace formé des applications de $\mathbb{R}^{n}$ dans $V$ dont la matrice relative à $f$ est antisymétrique, respectivement symétrique. Le sous-espace $\mathscr{A}_{f} V$ est le sous-espace vertical pour la fibration $p$ et $\mathscr{S}_{f} V$ en est un supplémentaire naturel. La distribution $f \mapsto \mathscr{S}_{f} V$ est clairement $\mathrm{O}_{n}$-équivariante (la conjuguée d'une matrice symétrique par une matrice orthogonale est encore symétrique), donc peut être considérée comme la distribution horizontale d'une $\mathrm{O}_{n}$-connexion que nous baptiserons connexion naturelle sur le fibré $p$.

Rappelons quelques faits bien connus. L'espace $\mathscr{M} V$, qui s'identifie à l'espace symétrique $\mathbb{R ~ G l}_{n} / \mathrm{O}_{n}$, est muni naturellement d'une métrique riemannienne $\langle$, $\rangle$ qui, au point $g$ vaut $\left\langle k, k^{\prime}\right\rangle_{g}=\operatorname{Trace}\left(K_{g} \circ K_{g}^{\prime}\right)$. Cette métrique est complète à courbure non-positive: deux éléments $h$ et $h^{\prime}$ de $\mathscr{M} V$ sont contenus dans un sous-espace plat maximal (de dimension $n$ ) passant par $g \in \mathscr{M} V$ si et seulement si les endomorphismes associés $H_{g}$ et $H_{g}^{\prime}$ commutent.

Parmi les courbes joignant $g$ à $h$ contenues dans un sous-espace plat, nous trouvons la géodésique de $g$ à $h$ pour la métrique $\left\langle\right.$, >, qui s'écrit $t \mapsto g_{t}=g \cdot\left(H_{g}\right)^{t}$ (où $\left(H_{g}\right)^{t}$ désigne la matrice symétrique positive puissance $t$-ième de $H_{g}$ ), et le segment $t \mapsto(1-t) g+t h$.

Nous avons alors

Proposition 2. Soient $g, h \in \mathscr{M V}$. La transformation $b_{h}^{g}$ coïncide avec le transport horizontal dans $\mathscr{B} V$ relatif à la connexion naturelle au-dessus de toute courbe joignant $g \grave{a} h$ dans $\mathscr{B} V$ contenue dans un sous-espace plat de $\mathscr{M} V$ passant par $g$ et $h$.

Preuve. Le transport horizontal de $\mathscr{B}_{g} V$ à $\mathscr{B}_{h} V$ induit par toute courbe $t \mapsto g_{t}$ joignant $g$ à $h$ est obtenu en intégrant dans $\mathscr{B} V$ l'équation différentielle définie par

$$
\frac{d f_{t}}{d t}=-\frac{1}{2}\left(\frac{d G_{t}}{d t}\right)_{g_{t}} \circ f_{t}
$$


L'image de $f_{0} \in \mathscr{B}_{g} V$ est obtenue en prenant la solution au temps 1 de l'équation ayant $f_{0}$ pour condition initiale.

Déterminons ce relèvement horizontal dans le cas du segment pour lequel $\dot{g}_{t}=h-g$ est constant. L'équation différentielle à résoudre s'écrit donc

$$
\left(\frac{d f_{t}}{d t}\right) \circ f_{t}^{-1}=-\frac{1}{2}\left(H_{g}-I\right) \circ\left(I+t\left(H_{g}-I\right)\right)^{-1}
$$

qui admet $f_{t}=\left(I+t\left(H_{g}-I\right)\right)^{-1 / 2} \circ f_{0}$ pour solution issue de $f_{0} \in \mathscr{B}_{g} V$, et nous avons bien

$$
f_{1}=H_{g}^{-1 / 2} \circ f_{0}=b_{h}^{g}\left(f_{0}\right) .
$$

Pour montrer que, pour toute courbe $\sigma$ joignant $g$ à $h$ se trouvant entièrement dans le sous-espace plat contenant $g$ et $h$, le transport horizontal le long de $\sigma$ ne dépend que de $g$ et $h$, nous avons besoin du lemme suivant qui détermine la courbure de la connexion naturelle dans le fibré $p: \mathscr{B} V \rightarrow \mathscr{M} V$.

Lemme 3. Soient $k$ et $k^{\prime} \in T_{g} \mathscr{M} V$. La courbure de la connexion naturelle sur le fibré $p: \mathscr{B} V \rightarrow \mathscr{M} V$ est donnée par

$$
\Omega_{k, k^{\prime}}(f)=-\frac{1}{4}\left[K_{g}, K_{g}^{\prime}\right] \circ f .
$$

Preuve. Nous devons identifier l'opposé de la partie verticale (grâce à la projection que définit la connexion) du crochet des relèvements horizontaux de deux champs de vecteurs définis localement au voisinage de $g$ et étendant $k$ et $k^{\prime}$. Comme $\mathscr{M} V$ est un ouvert d'un espace vectoriel, nous prenons comme extensions de $k$ et $k^{\prime}$ des champs constants dans $S^{2} V^{*}$ que nous notons encore $k$ et $k^{\prime}$. Le relèvement horizontal de $k$ en un repère $g$-orthonormé $f$ est $-\frac{1}{2} K_{g} \circ f$. Nous avons déjà remarqué précédemment que la courbe intégrale issue d'un repère $f_{0} \in \mathscr{B}_{g} V$ du relevé horizontal du champ linéaire constant $k$ dans $\mathscr{M} V$ s'écrit $f_{t}=\left(I+t K_{g}\right)^{-1 / 2} \circ f_{0}$. Nous pouvons calculer le crochet de Lie que nous cherchons comme le terme rectangle du deuxième ordre dans l'expression donnant l'extrémité de la courbe obtenue en parcourant successivement les courbes intégrales des relevés horizontaux de $k$ et $k^{\prime}$ pendant les temps $t, s,-t$ et $-s$. Cette expression est

$$
\left(I-s K_{g+s k^{\prime}}^{\prime}\right)^{-1 / 2} \circ\left(I-t K_{g+t k+s k^{\prime}}\right)^{-1 / 2} \circ\left(I+s K_{g+t k}^{\prime}\right)^{-1 / 2} \circ\left(I+t K_{g}\right)^{-1 / 2} \circ f_{0}
$$

dont le terme en st se calcule facilement en tenant compte des identités

$$
\begin{aligned}
K_{g+t k}^{\prime} & =\left(I+t K_{g}\right)^{-1} \circ K_{g}^{\prime}, \\
K_{g+t k+s k^{\prime}} & =\left(I+t K_{g+s k^{\prime}}\right)^{-1} \circ\left(I+s K_{g}^{\prime}\right)^{-1} \circ K_{g}, \\
K_{g+s k^{\prime}}^{\prime} & =\left(I+s K_{g}^{\prime}\right)^{-1} \circ K_{g}^{\prime} .
\end{aligned}
$$

En ne retenant que les termes du premier ordre et les termes en st dans le développement, nous obtenons

$$
\begin{aligned}
\left(I-s K_{g+s k^{\prime}}^{\prime}\right)^{-1 / 2} & \sim I+\frac{1}{2} s K_{g}^{\prime} \\
\left(I-t K_{g+t k+s k^{\prime}}\right)^{-1 / 2} & \sim I+\frac{1}{2} t K_{g}-\frac{1}{2} s t K_{g}^{\prime} \circ K_{g} \\
\left(I+s K_{g+t k}^{\prime}\right)^{-1 / 2} & \sim I-\frac{1}{2} K_{g}^{\prime}+\frac{1}{2} s t K_{g} \circ K_{g}^{\prime} \\
\left(I+t K_{g}\right)^{-1 / 2} & \sim I-\frac{1}{2} t K_{g}
\end{aligned}
$$


d'où la formule finale $\Omega_{k, k^{\prime}}(f)=-\frac{1}{4}\left[K_{g}, K_{g}^{\prime}\right] \circ f_{0}$ (noter que c'est bien un vecteur vertical puisque $\left[K_{g}, K_{g}^{\prime}\right]$ est antisymétrique).

Suite de la Preuve de la Proposition 2. Nous sommes maintenant dans une situation bien connue. Si deux courbes joignant $g$ à $h$ sont toutes deux tracées dans le sous-espace plat de $\mathscr{M} V$ contenant $g$ et $h$, on peut les voir comme les trajectoires entre les temps 0 et 1 de champs de vecteurs sur $\mathscr{M} V$. Les relevés horizontaux dans $\mathscr{B} V$ de ces champs de vecteurs ont pour crochets le relevé horizontal de ces crochets, et leurs flots vérifient les mêmes relations que leurs projections dans $\mathscr{M} V$. Leurs trajectoires vont donc aussi se rencontrer au temps 1.

Corollaire 4. Soient $g$, het $k \in \mathscr{M} V$ trois métriques appartenant au même sous-espace plat de $\mathscr{M} V$. Alors $b_{k}^{g}=b_{k}^{h} \circ b_{h}^{g}$.

Preuve. Il suffit de prendre pour courbe joignant $g$ à $k$ une courbe passant par $h$ et d'appliquer la Proposition 2.

On peut aussi vérifier directement que, comme $\left[H_{g}, K_{g}\right]=0$, nous avons $H_{k}^{-1 / 2} \circ K_{g}^{-1 / 2}=\left(H_{g} \circ K_{g}\right)^{-1 / 2}$.

La Proposition 2 qui est une version géométrique de la Proposition 1 s'étend directement au cadre spinoriel. Pour ce faire, choisissons une réalisation $\tilde{\mathscr{B}} V \mathrm{du}$ revêtement universel (i.e. à deux feuillets) de $\mathscr{B} V$. Chaque fibre $\mathscr{B}_{g} V$ est revêtue non trivialement par une variété $\widetilde{\mathscr{B}}_{g} V$ difféomorphe au groupe $\mathrm{Pin}_{n}$ que nous appellerons l'espace des bases spinorielles de $V$ (relatif à $g$ et au revêtement $\tilde{\mathscr{B}} V$ ).

Proposition 5. La transformation naturelle $b$, qui à tout couple de métriques $g$ et $h$ associe le difféomorphisme $b_{h}^{g}$ de $\mathscr{B}_{g} V$ sur $\mathscr{B}_{h} V$, peut être relevée en une transformation naturelle $\beta$ de l'espace des bases spinorielles $\tilde{\mathscr{B}} V$, qui à tout couple de métriques $g$ et $h$ associe un difféomorphisme $\operatorname{Pin}_{n}$-équivariant de $\tilde{\mathscr{B}}_{g} V$ sur $\tilde{\mathscr{B}}_{h} V$.

Preuve. La distribution horizontale $f \mapsto \mathscr{S}_{f} V$ se relève naturellement dans $\tilde{\mathscr{B}} V$ en une distribution horizontale déterminant une connexion $\operatorname{Pin}_{n}$-équivariante sur $\tilde{\mathscr{B}} V$, considéré comme un fibré $\operatorname{Pin}_{n}$-principal sur $\mathscr{M} V$.

La transformation $\beta$ est alors définie comme le transport parallèle dans le fibré $\operatorname{Pin}_{n}$-principal $\tilde{\mathscr{B}} V$ au-dessus de toute courbe joignant $g$ à $h$ contenue dans un sous-espace plat.

Remarque. Les considérations précédentes ne peuvent s'étendre telles quelles au cas des métriques lorentziennes $l$ sur un espace vectoriel $V$ de dimension $n+1$ pour plusieurs raisons que nous indiquons maintenant.

Dans ce cas on doit utiliser la métrique de Minkowski $m=\left(\varepsilon^{1}\right)^{2}+\cdots+\left(\varepsilon^{n}\right)^{2}-$ $\left(\varepsilon^{n+1}\right)^{2}$ comme modèle sur $\mathbb{R}^{n+1}$ (en notant $\left(\varepsilon^{i}\right)$ la base duale de la base canonique). L'application $l \mapsto q(f)=\left(f^{-1}\right)^{*} m$ définit encore une fibration $q: \mathscr{B} V \rightarrow \mathscr{L} V$ où $\mathscr{L} V$ désigne l'espace des métriques lorentziennes sur $V$ qui est un cône ouvert (non convexe!) de $S^{2} V^{*}$. Le sous-espace vertical en $f \in \mathscr{B} V$ s'identifie naturellement aux applications $f \circ B$ de $\mathbb{R}^{n+1}$ dans $V$ où $B$ désigne une matrice dans l'algèbre de Lie du groupe de Lorentz $\mathrm{O}_{n, 1}$.

Il y a encore une distribution horizontale $\mathrm{O}_{n, 1}$-invariante déterminée par les éléments symétriques au sens lorentzien permettant de déterminer un transport parallèle au-dessus de n'importe quelle courbe. Mais en général il n'est pas possible de distinguer une famille de courbes privilégiées joignant deux éléments quelconques 
de $\mathscr{L} V$. En particulier l'interpolation linéaire ne permet pas de joindre deux éléments quelconques qui ne sont pas non plus joints par une géodésique pour la métrique naturelle $\langle$,$\rangle qui s'écrit encore \left\langle k, k^{\prime}\right\rangle_{l}=\operatorname{Trace}\left(K_{l} \circ K_{l}^{\prime}\right)$ (noter que cette métrique n'est pas riemannienne mais de signature $\left.\left(\frac{1}{2}\left(n^{2}+n+2\right), n\right)\right)$.

De plus il n'est pas clair que l'on puisse donner la formule de la transformation naturelle entre deux métriques lorentziennes $l$ et $k$ car, si l'automorphisme $K_{l}=l^{-1} \cdot k$ est bien défini, il n'a pas nécessairement toutes ses valeurs propres positives. Il n'est donc pas possible d'en prendre la racine carrée sans faire des choix de coupure dans le plan complexe ce qui créera des difficultés dans le cas à paramètres qui est notre véritable but.

Cependant, ces difficultés disparaissent lorsqu'on reste au voisinage d'une métrique lorentzienne donnée. Ceci suffit pour étendre au cas lorentzien la construction d'une dérivée de Lie métrique donnée dans la Sect. II.

\section{Dérivation de Lie Métrique des Champs de Tenseurs et de Spineurs}

La dérivée de Lie usuelle, que nous noterons $\mathscr{L}_{X}$, est fondée sur la possibilité de transporter les tenseurs le long du flot $\left(\xi_{t}\right)$ d'un champ de vecteurs (quelconque) $X$. Cette possibilité n'existant pas pour les champs de spineurs, nous lui substituons, via les transformations $b_{\xi_{t}^{*} g}^{g}$ et $\beta_{\tilde{\xi}_{t}^{*} \gamma}^{\gamma}$ déduits des constructions des paragraphes précédents, une dérivée de Lie métrique $\mathscr{L}_{X}^{g}$ agissant sur les champs de tenseurs et les champs de spineurs.

Comme la dérivée de Lie usuelle, $\mathscr{L}_{X}^{g}$ est une dérivation pour le produit tensoriel. Contrairement à la dérivée usuelle, elle préserve la métrique $g$, i.e., pour tout champ de vecteurs $X$, on a $\mathscr{L}_{X}^{g} g=0$ (voir Proposition 15), mais elle possède une courbure (voir Proposition 18) en ce sens que $\mathscr{L}_{[X, Y]}^{g}$ diffère en général du commutateur $\left[\mathscr{L}_{X}^{g}, \mathscr{L}_{Y}^{g}\right]$. Les deux dérivations $\mathscr{L}_{X}$ et $\mathscr{L}_{X}^{g}$ agissant sur les champs de tenseurs coïncident si et seulement si le champ de vecteurs $X$ est de Killing, i.e. si son flot est formé d'isométries. La dérivée de Lie métrique agissant sur les champs de spineurs coïncide avec la dérivée de Lie introduite par des voies différentes par Y. Kosmann (cf. [14]).

Nous commençons par étendre les notions algébriques introduites précédemment au cadre des variétés différentielles et des champs.

Sur une variété différentielle $M$ de dimension $n$, nous introduisons le fibré principal sous le groupe $\mathbb{R} \mathrm{Gl}_{n}$ de ses repères linéaires que nous notons $\mathscr{B} M$ et, pour une métrique riemannienne $g$ sur $M$, le fibré principal sous le groupe $\mathrm{O}_{n}$ des repères $g$-orthonormées que nous notons $O_{g} M$. Sa fibre en un point $m$ n'est rien d'autre que $\mathscr{B}_{g_{m}}\left(T_{m} M\right)$ avec les notations de la Sect. I Soit $h$ une autre métrique riemannienne sur $M, O_{h} M$ le $\mathrm{O}_{n}$-fibré principal des repères $h$-orthonormés. Nous noterons $b_{h}^{g}$ le morphisme $\mathrm{O}_{n}$-équivariant de $O_{g} M$ sur $O_{h} M$ dont la restriction à chaque fibre en un point $m$ de $M$ est égale à $b_{h_{m}}^{g_{m}}$.

Pour toute représentation $\mu$ de $\mathrm{O}_{n}$ sur un espace vectoriel $E$, nous noterons de même $b_{h}^{g}$ l'isomorphisme de fibrés vectoriels de $E_{g} M=O_{g} M \times{ }_{\mu} E$ sur $E_{h} M=$ $O_{h} M \times{ }_{\mu} E$ défini comme suit: à tout élément $\xi$ de $E_{g} M$, de composante $u$ dans $E$ relativement à un repère $g$-orthonormé $f$, nous associons l'élément de $E_{h} M$ ayant même composante $u$ relativement au repère $h$-orthonormé $b_{h}^{g}(f)$, autrement dit

$$
b_{h}^{g}([f, u])=\left[b_{h}^{g}(f), u\right] .
$$


L'équivariance de $b_{h}^{g}$ de $O_{g} M$ dans $O_{h} M$ implique que $b_{h}^{g}$ est bien défini de $E_{g} M$ dans $E_{h} M$.

Remarque 6. Lorsque $\mu$ est la restriction à $\mathrm{O}_{n}$ d'une représentation linéaire du groupe linéaire $\mathbb{R ~ G l}_{n}$ également notée $\mu$, les deux fibrés vectoriels $E_{g} M$ et $E_{h} M$ s'identifient naturellement l'un et l'autre au fibré de tenseurs $E M=\mathscr{B} M \times_{\mu} E$, mais $b_{h}^{g}$, vu comme un endomorphisme de EM, ne coïncide pas en général avec l'identité. En particulier, si $\mu$ est la représentation tautologique $\mu_{0}$ de $\mathrm{O}_{n}$ sur $\mathbb{R}^{n}$, les fibrés $O_{g} M \times{ }_{\mu_{0}} \mathbb{R}^{n}$ et $O_{h} M \times{ }_{\mu_{0}} \mathbb{R}^{n}$ sont naturellement identifiés au fibré tangent $T M$ et $b_{h}^{g}$ coïncide dans ce cas avec $H_{g}^{-1 / 2}$, l'inverse de la racine carrée positive de $H_{g}=g^{-1} \cdot h$ qui est une isométrie de $(T M, g)$ sur $(T M, h)$.

Considérons le cas où la variété $M$ est orientée et spin (autrement dit nous supposons que ses deux premières classes de Stiefel-Whitney $w_{1}(M) \in H^{1}(M, \mathbb{Z} / 2 \mathbb{Z})$ et $w_{2}(M) \in H^{2}(M, \mathbb{Z} / 2 \mathbb{Z})$ sont nulles, cf. [17]). Nous notons $\mathbb{R} \widetilde{\mathrm{G}} l_{n}^{+}$le revêtement universel du groupe linéaire positif $\mathbb{R ~ G l}_{n}^{+}$.

Choisissons une structure spinorielle $\alpha$ sur $M$ (c'est un point dans un certain espace affine modelé sur le $\mathbb{Z} / 2 \mathbb{Z}$-espace vectoriel $H^{1}(M, \mathbb{Z} / 2 \mathbb{Z})$ ) que nous réalisons par un fibré $\mathbb{R} \tilde{\mathrm{G}} \mathrm{l}_{n}^{+}$-principal noté $\mathscr{\mathscr { B }}^{+} M$ revêtant non trivialement en chaque point $m$ de $M$ le fibré $\mathscr{B}_{m}^{+} M$ des repères linéaires positifs en ce point. Le choix de $\widetilde{\mathscr{B}}^{+} M$ détermine pour chaque métrique $g$ une métrique spinorielle $\gamma$ sous la forme d'un fibré $\operatorname{Spin}_{n}$-principal, à savoir le sous-fibré $\operatorname{Spin}_{\gamma} M$ de $\mathscr{B}^{+} M$ qui revêt le fibré $S O_{g} M$ des repères $g$-orthonormés positifs.

Pour toute représentation linéaire (réelle ou complexe) $\sigma$ du groupe $\operatorname{Spin}_{n}$ dans $\Sigma$, nous notons $\Sigma_{\gamma} M=\operatorname{Spin}_{\gamma} M \times_{\sigma} \Sigma$ le fibré vectoriel associé. Nous dirons que $\sigma$ est une représentation spinorielle lorsqu'elle est la restriction d'une représentation de l'algèbre de Clifford que nous supposerons unitaire, en ce sens que l'image de tout vecteur unitaire est unitaire. Nous donnerons alors le nom de fibré de spineurs au fibré vectoriel hermitien correspondant. Par définition, tout fibré de spineurs est donc un module à gauche pour le fibré en algèbres de Clifford $\mathrm{Cl}_{g} M$.

La transformation naturelle $\beta$ définie dans le cadre algébrique s'étend différentiablement au fibré des bases spinorielles, et par suite détermine une famille d'homomorphismes entre fibrés de spineurs pour différentes métriques que nous notons encore $\beta$. Précisément, si $\gamma$ et $\eta$ sont deux métriques spinorielles correspondant au même fibré $\tilde{\mathscr{B}}^{+} M$, alors, à tout $\gamma$-spineur $\psi$ de composante $v$ dans une base spinorielle $\phi$, nous associons comme précédemment le $\eta$-spineur $\beta_{\eta}^{\gamma}(\psi)$ de même composante $v$, autrement dit

$$
\beta_{n}^{\gamma}([\phi, v])=\left[\beta_{\eta}^{\gamma}(\phi), v\right] .
$$

A nouveau, cette correspondance est bien définie à cause de la $\operatorname{Spin}_{n}$-équivariance de la transformation naturelle $\beta_{\eta}^{\gamma}$. Lorsque $\sigma$ est une représentation spinorielle, la transformation $\beta_{\eta}^{\gamma}$ est compatible avec la multiplication de Clifford en ce sens que, pour toute forme extérieure $\omega$ vue comme un élément de $\mathrm{Cl}_{g} M$ et tout $\gamma$-spineur $\psi$, on a

$$
\beta_{\eta}^{\gamma}\left(\omega_{\cdot \gamma} \psi\right)=b_{h}^{g}(\omega)_{\cdot \eta} \beta_{n}^{\gamma}(\psi)
$$

Tout difféomorphisme $\xi$ de $M$ se relève en un difféomorphisme $\mathrm{O}_{n}$-équivariant $\xi^{O}$ de $O_{g} M$ en posant

$$
\xi^{O}=b_{g}^{\left(\xi^{-1}\right)^{*} g} \circ T \xi
$$


Un tel relèvement existe aussi dans le cas spinoriel dans $\operatorname{Spin}_{\gamma} M$ à condition que $\xi$ préserve à la fois l'orientation et la structure spinorielle $\alpha$. Mais ce relèvement n'est défini a priori qu'à l'action près de $\mathbb{Z} / 2 \mathbb{Z}$ (cf. [14]). Toutefois, le relèvement est défini sans ambiguïté si le difféomorphisme est relié par un chemin à l'identité. Nous le notons alors $\xi^{\text {Spin }}$.

Cela nous permet de proposer la définition suivante pour la dérivée de Lie métrique d'un champ (local) de repères orthonormés.

Définition 7. Soit $X$ un champ de vecteurs (quelconque) défini sur une variété riemannienne $(M, g)$ dont le flot local est noté $\left(\xi_{t}\right)$. La g-dérivée de Lie d'un champ de repères $g$-orthonormés $F$ par rapport à $X$ en un point $x$ est l'élément $\mathscr{L}_{X}^{g} F$ de l'algèbre de Lie $\mathfrak{D}_{n}$ de $\mathrm{O}_{n}$ défini par

$$
\mathscr{L}_{X}^{g} F=\left.\frac{d}{d t}\left(\xi_{-t}^{o} \circ F \circ \xi_{t}\right)\right|_{t=0}
$$

via l'identification canonique de l'espace tangent vertical au point $f(x)$ avec $\mathfrak{D}_{n}$.

Si $M$ est munie d'une métrique spinorielle $\gamma$, la $\gamma$-dérivée de Lie d'un champ de $\gamma$-repères spinoriels $\Phi$ par rapport à X est l'élément $\mathscr{L}_{X}^{\gamma} \Phi$ de l'algèbre de Lie $\Xi_{p i n}$ du groupe $\operatorname{Spin}_{n}$

$$
\mathscr{L}_{X}^{\gamma} \Phi=\left.\frac{d}{d t}\left(\tilde{\xi}_{-t}^{\operatorname{Spin}_{0}} \Phi \circ \xi_{t}\right)\right|_{t=0}
$$

Lorsque le repère spinoriel $\Phi$ est un relèvement $\tilde{F}$ d'un repère orthonormé $F$, la dérivée de Lie métrique $\mathscr{L}_{X}^{\gamma} \tilde{F}$ est égale à l'image de $\mathscr{L}_{X}^{g} F$ par l'isomorphisme de $\operatorname{Spin}_{n}$ sur $\mathfrak{S D}_{n}$ induit par la projection naturelle de $\operatorname{Spin}_{n}$ sur $S O_{n}$. Dans les identifications classiques de $\mathfrak{S p i n}_{n}$ et de $\mathfrak{S}_{\mathfrak{D}_{n}}$ comme espaces vectoriels avec l'espace $\mathscr{A} \mathbb{R}^{n}$ des endomorphisms antisymétriques de $\mathbb{R}^{n}$, on a alors

$$
\mathscr{L}_{X}^{\gamma} \tilde{F}=\frac{1}{2} \mathscr{L}_{X}^{g} F
$$

Proposition 9. Pour un champ de repères orthonormés $F$, la dérivée de Lie métrique $\mathscr{L}_{X}^{g} F$ et la dérivée de Lie ordinair $\mathscr{L}_{X} F$ (vue comme un élément de $\left(\mathfrak{b l}_{n}\right.$ ) sont reliées par la formule

$$
\mathscr{L}_{X}^{g} F=A\left(\mathscr{L}_{X} F\right)
$$

où A désigne la projection naturelle de $\mathfrak{G I}_{n}$ sur $\mathfrak{D}_{n}$ qui à une matrice associe sa partie antisymétrique.

Preuve. La dérivée de Lie métrique $\mathscr{L}_{X}^{g} F$ s'écrit encore

$$
\mathscr{L}_{X}^{g} F=T F(X)-X_{F(m)}^{o},
$$

où $X^{O}$ désigne le relevé métrique sur $O_{g} M$ du champ de vecteurs $X$ défini en le repère $f$ au point $m$ par

$$
X_{f}^{o}=\left.\frac{d}{d t}\left(b_{g}^{\xi_{-}^{*}{ }^{g}}\left(T \xi_{t} \circ f\right)\right)\right|_{t=0} .
$$

De la même manière, $\mathscr{L}_{X} F$ s'écrit

$$
\mathscr{L}_{X} F=T F(X)-X_{F(m)}^{G l},
$$


où $X^{G l}$ est le relevé naturel de $X$ sur $G l M$ défini, en $f$, par

$$
X_{f}^{G l}=\left.\frac{d}{d t}\left(T_{m} \xi_{t} \circ f\right)\right|_{t=0} .
$$

Lemme 11. En tout point $f$ de $O_{g} M \subset G l M$, on a

$$
X_{f}^{G l}-X_{f}^{o}=\frac{1}{2}\left(\mathscr{L}_{X} g\right)_{f}
$$

où $\left(\mathscr{L}_{X} g\right)_{f}$ est l'élément (symétrique) de $\mathrm{Gl}_{n}$ déterminé par $\mathscr{L}_{X} g$ via le repère $f$.

Preuve. Il suit des définitions des relevés $X^{o}$ et $X^{G l}$ que

$$
X_{f}^{o}-X_{f}^{G l}=\left(\left.\frac{d}{d t} b_{g}^{\xi_{-t}^{*} g}\right|_{t=0}\right)_{f}
$$

en utilisant la notation de l'énoncé du Lemme 11.

Le résultat suit directement de la Remarque 6.

La Proposition 9 résulte immédiatement des formules (10) et $\left(10^{\prime}\right)$, du Lemme 11 et du fait que $\frac{1}{2}\left(\mathscr{L}_{X} g\right)_{F}$ est la partie symétrique de $\mathscr{L}_{X} F$ comme on le voit directement en utilisant la fait que $F$ est une base orthonormée en chaque point.

Proposition 12. Pour toute fonction a (respectivement $\alpha$ ) de $M$ à valeurs dans $\mathrm{O}_{n}$ (respectivement $\operatorname{Spin}_{n}$ ), on a

$$
\begin{aligned}
& \mathscr{L}_{X}^{g}(F \cdot a)=\left(\operatorname{Ad} a^{-1}\right)\left(\mathscr{L}_{X}^{g} F\right)+a^{-1}\left(\mathscr{L}_{X} a\right), \\
& \mathscr{L}_{X}^{\gamma}(\Phi \cdot \alpha)=\left(\operatorname{Ad} \alpha^{-1}\right)\left(\mathscr{L}_{X}^{\gamma} \Phi\right)+\alpha^{-1}\left(\mathscr{L}_{X} \alpha\right) .
\end{aligned}
$$

Preuve. La formule (12) est une conséquence immédiate de la Proposition 9 et de la formule analogue pour la dérivation de Lie ordinaire.

La formule $\left(12^{\prime}\right)$ résulte de la formule (8).

Ayant défini la dérivée de Lie métrique d'un champ de repères, nous définissons la dérivée de Lie métrique des champs de tenseurs et de spineurs de la manière naturelle suivante.

Définition 13. Soit $E_{\mu} M$ le fibré vectoriel déterminé, via $O_{g} M$, par une représentation linéaire $\mu$ de $\mathrm{O}_{n}$. Pour tout champ de vecteurs $X$, la dérivée de Lie métrique $\mathscr{L}_{X}^{g} S$ d'une section $S$ de $E_{\mu} M$ dans la direction de $X$ est définie par

$$
\mathscr{L}_{X}^{g} S=\left(F, \mathscr{L}_{X} u+T \mu\left(\mathscr{L}_{X}^{g} F\right) u\right)
$$

où u est la composante de $S$ (dans E) relativement à une section locale $F$ de $O_{g} M$.

Soit $\Sigma_{\gamma} M$ le fibré vectoriel déterminé, via $\operatorname{Spin}_{\gamma} M$, par une représentation linéaire $\sigma$ de $\operatorname{Spin}_{n}$. La dérivée de Lie métrique $\mathscr{L}_{X}^{\gamma} \psi$ d'une section $\psi$ de $\Sigma_{\gamma} M$ dans la direction de $X$ est définie par

$$
\mathscr{L}_{X}^{\gamma} \psi=\left(\Phi, \mathscr{L}_{X} v+T \sigma\left(\mathscr{L}_{X}^{\gamma} \Phi\right) v\right)
$$

où v est la composante de $\psi$ (dans $\Sigma$ ) relativement à une section locale $\Phi$ de $\operatorname{Spin}_{\gamma} M$.

Note. Dans les formules précédentes, $T \mu$ et $T \sigma$ désignent les dérivées en l'identité des représentations $\mu$ et $\sigma$.

Remarques. i) Il résulte immédiatement de la Proposition 12 que la définition 13 ne dépend pas du choix du repère local. 
ii) Lorsque la variété est spinorielle, tous les fibrés de tenseurs peuvent être construits à partir du fibré des repères spinoriels (mais ne sont pas des fibrés de spineurs!), et les deux définitions de la dérivation de Lie métrique coïncident.

iii) Comme la dérivée de Lie ordinaire, la dérivée de Lie métrique est une dérivation pour le produit tensoriel de tenseurs qui coïncide avec la dérivée usuelle sur les scalaires. Il en résulte immédiatement que la dérivée de Lie métrique est une dérivation pour le produit tensoriel des spineurs et/ou des tenseurs.

Proposition 14. La dérivée métrique $\mathscr{L}_{X}^{g}$ agissant sur un champ de $\mu$-tenseurs $S$ se déduit de la dérivation de Lie ordinaire par la formule

$$
\mathscr{L}_{X}^{g} S=\mathscr{L}_{X} S+\frac{1}{2}\left(\left(\mathscr{L}_{X} g\right)^{\#}\right)_{\cdot \mu} S,
$$

où $\left(\mathscr{L}_{X} g\right)^{\#}$ désigne l'endomorphisme associé par la métrique $g$ à $\mathscr{L}_{X} g$ et ${ }_{\cdot \mu}$ l'action d'un champ d'endomorphismes sur un champ de $\mu$-tenseurs par la représentation $\mu$.

En conséquence, les deux dérivations de Lie coïncident lorsque $X$ est un champ de Killing.

Preuve. Conséquence de la Proposition 9 compte tenu du Lemme 11.

Les propriétés essentielles de la dérivation de Lie métrique sont rassemblées dans les propositions qui suivent.

Proposition 15. La dérivée de Lie métrique $\mathscr{L}^{g}$ préserve la métrique g, i.e., pour tout champ de vecteurs $X$,

$$
\mathscr{L}_{X}^{g} g=0 .
$$

Plus généralement, pour toute représentation (orthogonale ou unitaire) $\mu$ de $\mathrm{O}_{n}$ et tout champ de $\mu$-tenseurs $S_{1}$ et $S_{2}$, nous avons pour tout champ de vecteurs $X$

$$
\mathscr{L}_{X}\left(S_{1}, S_{2}\right)_{g}=\left(\mathscr{L}_{X}^{g} S_{1}, S_{2}\right)_{g}+\left(S_{1}, \mathscr{L}_{X}^{g} S_{2}\right) g
$$

où $(,)_{g}$ désigne le produit scalaire euclidien (hermitien) déterminé par $\mu$.

Si $M$ est munie d'une métrique spinorielle $\gamma$ et si $\Sigma_{\gamma} M$ est le fibré vectoriel associé à une représentation unitaire $\sigma$ de $\operatorname{Spin}_{n}$, pour toutes sections $\psi_{1}$ et $\psi_{2}$ de $\Sigma_{\gamma} M$ nous avons

$$
\mathscr{L}_{X}\left(\psi_{1}, \psi_{2}\right)_{\gamma}=\left(\mathscr{L}_{X}^{\gamma} \psi_{1}, \psi_{2}\right)_{\gamma}+\left(\psi_{1}, \mathscr{L}_{X}^{\gamma} \psi_{2}\right)_{\gamma},
$$

où $(,)_{\gamma}$ désigne le produit scalaire euclidien ou hermitien défini sur $\Sigma_{\gamma} M$. Si $\sigma$ est une représentation spinorielle, pour toute forme différentielle extérieure $\omega$ (vue comme une section de $\mathrm{Cl}_{g} M$ agissant sur $\Sigma_{\gamma} M$ via la représentation $\sigma$ ), nous avons

$$
\mathscr{L}_{X}^{\gamma}\left(\omega_{\cdot \gamma} \psi\right)=\left(\mathscr{L}_{X}^{g} \omega\right)_{\cdot \gamma} \psi+\omega_{\cdot \gamma}\left(\mathscr{L}_{X}^{\gamma} \psi\right),
$$

où., désigne l'action de $\mathrm{Cl}_{g} M$ sur le fibré en spineurs $\Sigma_{\gamma} M$ (la représentation $\sigma$ est sous-entendue).

Preuve. Les formules (15) et $\left(15^{\prime}\right)$ sont des conséquences immédiates des formules de définition (13) et $\left(13^{\prime}\right)$. La nullité de $\mathscr{L}_{x}^{g} g$ s'obtient alors en considérant la représentation fondamentale de $\mathrm{O}_{n}$ dans $\mathbb{R}^{n}$.

La formule $\left(15^{\prime \prime}\right)$ se démontre ainsi: par définition d'une représentation spinorielle, $\sigma$ est la restriction au groupe $\operatorname{Spin}_{n}$ d'un homomorphisme linéaire (encore noté $\sigma$ ) de l'algèbre de Clifford $\mathrm{Cl}_{n}$ dans End $\Sigma$. La multiplication de Clifford d'un champ de $\gamma$-spineurs $\psi$ par une forme différentielle extérieure $\omega$ s'écrit 
relativement à un repère $\gamma$-spinoriel $F$ relevé d'un repère $g$-orthonormé $F$ par la formule $\omega \cdot \psi=(\tilde{F}, \sigma(\alpha)(v))$ où $\alpha$ est l'expression de $\omega$ dans $\mathrm{Cl}_{n}$ relativement à $F$ et $v$ celle de $\psi$ relativement à $\widetilde{F}$. On a donc, d'après la formule de définition $\left(13^{\prime}\right)$,

$$
\begin{aligned}
\mathscr{L}_{X}^{\gamma}(\omega . \psi) & =\left[\tilde{F}, \mathscr{L}_{X}(\sigma(\alpha) v)+T \sigma\left(\mathscr{L}_{X}^{\gamma} \tilde{F}\right)(\sigma(\alpha)(v))\right] \\
& =\left[\tilde{F}, \sigma\left(\mathscr{L}_{X} \alpha\right)(v)+\sigma(\alpha)\left(\mathscr{L}_{X} v\right)+\sigma\left(\operatorname{ad}\left((T \sigma)\left(\mathscr{L}_{X}^{\gamma} \tilde{F}\right)\right) \alpha\right)(v)+\sigma(\alpha)\left(\sigma\left(\mathscr{L}_{X}^{\gamma} \tilde{F}\right)(v)\right]\right) \\
& =\left[\tilde{F}, \sigma\left(\left(\mathscr{L}_{X} \alpha\right)+\operatorname{ad}\left((T \sigma)\left(\mathscr{L}_{X}^{\gamma} \tilde{F}\right)\right) \alpha\right)(v)\right]+\left[\tilde{F}, \sigma(\alpha)\left(\mathscr{L}_{X} v+(T \sigma)\left(\mathscr{L}_{X}^{\gamma} \tilde{F}\right)(v)\right)\right] \\
& =\left(\mathscr{L}_{X}^{g}(\omega) \cdot \psi+\omega \cdot \mathscr{L}_{X}^{\gamma} \psi .\right.
\end{aligned}
$$

Proposition 16. Sur une variété riemannienne $(M, g)$, soient a une fonction, $X$ un champ de vecteurs et $S$ un champ de $\mu$-tenseurs. Alors

$$
\mathscr{L}_{a X}^{g} S=a \mathscr{L}^{g} S-\frac{1}{2}\left(d a \wedge X^{b}\right)_{\cdot \mu} S .
$$

Si $M$ est munie d'une métrique spinorielle $\gamma$, et si $\Sigma_{\gamma} M$ est le fibré vectoriel associé $\grave{a}$ une représentation $\sigma$ de $\operatorname{Spin}_{n}$, pour toute section $\psi$ de $\Sigma_{\gamma} M$, nous avons

$$
\mathscr{L}_{a X}^{\gamma} \psi=a \mathscr{L}^{\gamma}{ }^{\gamma} \psi-\frac{1}{4}\left(d a \wedge X^{b}\right)_{\cdot \gamma} \psi .
$$

Preuve. La proposition est une conséquence directe de la formule de définition (13) compte-tenu de la Proposition 9 et dans le cas spinoriel de la formule (8).

Nous pouvons comparer la dérivation de Lie métrique avec la connexion de Levi-Civita. Pour cela rappelons que la connexion de Levi-Civita $D^{g}$ associée à la métrique $g$ se relève au fibré des repères spinoriels. Nous notons naturellement $D^{\gamma}$ la connexion ainsi induite, dont on déduit une dérivation covariante pour les champs de $\gamma$-spineurs. La seule chose à laquelle il faut prendre garde est que l'action de l'algèbre extérieure par multiplication de Clifford fait apparaître un facteur $\frac{1}{2}$ dans l'action des formes de connexion (voir (8)). Nous avons alors

Proposition 17. La dérivation de Lie métrique et la dérivation covariante de LeviCivita sont reliées pour des champs de vecteurs $X$ et de $\mu$-tenseurss par la formule

$$
\mathscr{L}_{X}^{g} S=D_{X}^{g} S-\frac{1}{2}\left(d X^{b}\right) \cdot{ }_{\gamma} S
$$

(où $X^{b}$ désigne la 1-forme duale de $X$ pour la métrique g et d la différentielle extérieure), et pour un champ de $\gamma$-spineurs $\psi$ par la formule

$$
\mathscr{L}_{X}^{\gamma} \psi=D_{X}^{\gamma} \psi-\frac{1}{4}\left(d X^{b}\right)_{\cdot \gamma} \psi
$$

Preuve. Les formules (17) et $\left(17^{\prime}\right)$ se vérifient aisément à partir de la formule de définition (13) et la formule analogue pour la dérivation covariante. Elles peuvent aussi se déduire de la Proposition 16 par l'argument universel suivant. D'après la Proposition 15, l'application $a \mapsto \mathscr{L}_{a X}^{g}+\frac{1}{4}\left(d a \wedge X^{b}\right)_{\cdot \mu}$ est un opérateur différentiel d'ordre 0 en $a$. Il en est de même de $a \mapsto D_{a X}^{g}$. La différence des deux membres de (15) est donc tensorielle en $X$. Il suffit alors de constater que cette 1 -forme est un invariant $g$-orthogonal, donc en fait la forme nulle.

Proposition 18. La courbure de la g-dérivation de Lie, i.e. son défaut d'être un homomorphisme d'algèbres de Lie entre l'algèbre de Lie $\mathscr{T} M$ des champs de vecteurs sur $M$ et l'algèbre des opérateurs différentiels sur les champs de vecteurs, est donnée par la formule

$$
\left[\mathscr{L}_{X}^{g}, \mathscr{L}_{Y}^{g}\right]-\mathscr{L}_{[X, Y]}^{g}=-\frac{1}{4}\left[\left(\mathscr{L}_{X} g\right)^{\#},\left(\mathscr{L}_{Y} g\right)^{\#}\right]
$$


Preuve. C'est une vérification directe à partir de la Proposition 9 en utilisant que la partie antisymétrique du commutateur de deux endomorphismes est la somme du commutateur des parties antisymétriques et du commutateur des parties symétriques.

Remarque. Le membre de droite de la formule (11) est nul dès que $X$ (ou $Y$ ) est une transformation infinitésimale conforme, car alors $\left(\mathscr{L}_{X} g\right)^{\#}\left(\right.$ ou $\left.\left(\mathscr{L}_{Y} g\right)^{\#}\right)$ est un multiple de l'identité. A cause de cela, on peut considérer que l'expression "dérivation de Lie" utilisée pour désigner $\mathscr{L}_{X}^{g}$ n'est pleinement justifiée que pour ces champs de vecteurs et on revient à la remarque faite p. 101 de [19].

\section{Variations de l'Opérateur de Dirac et de ses Valeurs Propres par Changement de Métrique}

Rappelons que, pour tout champ $\psi$ de $\gamma$-spineurs (relatif à une représentation spinorielle $\sigma$ quelconque),

$$
\mathscr{D}^{\gamma} \psi=\sum_{i=1}^{n} f_{i \cdot \gamma} \mathscr{D}_{f_{i}}^{\gamma} \psi
$$

où $\left(f_{i}\right)$ est une base $g$-orthonormée au point considéré.

Maintenant que nous savons comparer les champs de spineurs pour deux métriques spinorielles $\gamma$ et $\eta$ (correspondant à la même classe spinorielle, mais à deux métriques $g$ et $h$ distinctes), il est possible de comparer les opérateurs de Dirac $\mathscr{D}^{\gamma}$ et $\mathscr{D}^{\eta}$ agissant respectivement sur les champs de $\gamma$-spineurs et de $\eta$-spineurs en introduisant l'opérateur transmué défini par

$$
{ }^{\gamma} \mathscr{D}^{\eta}=\left(\beta_{\eta}^{\gamma}\right)^{-1} \circ \mathscr{D}^{\eta} \circ \beta_{\eta}^{\gamma} \text {. }
$$

Théorème 20. Le transmué ${ }^{\gamma} \mathscr{D}^{\eta} d u$ $\eta$-opérateur de Dirac a, pour tout champ $\psi$ de $\gamma$-spineurs, l'expression suivante

$$
\begin{aligned}
{ }^{\gamma} \mathscr{D}^{\eta} \psi= & \sum_{i=1}^{n} f_{i \cdot \gamma} D_{H_{g}^{-1 / 2}\left(f_{i}\right)}^{\gamma} \psi \\
& +\frac{1}{2} \sum_{i=1}^{n} f_{i \cdot \gamma} H_{g}^{1 / 2}\left(D_{H_{g}^{-1 / 2}\left(f_{i}\right)}^{g} H_{g}^{-1 / 2}+{ }^{g} A_{H_{g}^{-1 / 2}\left(f_{i}\right)}^{h}{ }^{\circ} H_{g}^{-1 / 2}\right)_{\cdot \gamma} \psi,
\end{aligned}
$$

où $\left(f_{i}\right)$ est un repère g-orthonormé et où ${ }^{g} A^{h}=D^{h}-D^{g}$ est la différence des deux connexions de Levi-Civita de $h$ et $g$ respectivement.

Preuve. Par définition-même du transmué, on a

$$
{ }^{\gamma} \mathscr{D}^{\eta}(\psi)=\sum_{i=1}^{n} f_{i \cdot \gamma}\left({ }^{\gamma} D_{H_{g}^{-1 / 2}\left(f_{i}\right)}^{\eta} \psi\right)
$$

où ${ }^{\gamma} D^{\eta}=\left(\beta_{\eta}^{\gamma}\right)^{-1} \circ D^{\eta} \circ\left(\beta_{\eta}^{\gamma}\right)$ est l'image sur $\operatorname{Spin}_{\gamma} M$ de la connexion de Levi-Civita $D^{\eta}$ par la transformation naturelle $\beta_{\eta}^{\gamma}$. Elle est en fait induite par la connexion ${ }^{g} D^{h}=H_{g}^{1 / 2} \circ D^{h} \circ H_{g}^{-1 / 2}$ définie sur $S O_{g} M$, transmuée de $D^{h}$ par la transformation $b_{h}^{g}$.

La formule (20) résulte d'un calcul direct utilisant la formule (6) et le fait que $\left(b_{h}^{g}\left(f_{i}\right)\right)$ est une base $h$-orthonormée (on rappelle que $b_{h}^{g}\left(f_{i}\right)=H_{g}^{-1 / 2}\left(f_{i}\right)$ ). 
Remarques. i) En observant le symbole principal de ${ }^{\gamma} \mathscr{D}^{\eta}$ donné par le terme principal du second membre de (20), on remarquera que cet opérateur n'est pas un opérateur de Dirac.

ii) Le terme complémentaire du second membre de (20) fait intervenir l'expression $H^{1 / 2}\left(D^{g} H^{-1 / 2}+{ }^{g} A^{h} \circ H^{-1 / 2}\right)$ qui est égale à la différence ${ }^{g} D^{h}-D^{g}$.

Comme la connexion ${ }^{g} D^{h}$ est $g$-métrique, $H^{1 / 2}\left(D_{X}^{g} H^{-1 / 2}+{ }^{g} A_{X}^{h} \circ H^{-1 / 2}\right)$ est antisymétrique pour tout $X$ et comme telle considérée comme un élément de l'algèbre de Clifford.

Pour étudier la dérivée de $\mathscr{D}^{\eta}$ quand $h$ varie dans l'espace des métriques $\mathscr{M} M$, nous substituons à $\mathscr{D}^{\eta}$ l'opérateur transmué ${ }^{\gamma} \mathscr{D}^{\eta}$ qui opère sur les sections du fibré (fixe!) des $\gamma$-spineurs.

Un vecteur tangent en une métrique $g$ à $\mathscr{M} M$ sera identifié à une forme bilinéaire symétrique $k$, par exemple le vecteur tangent en $t=0$ à la courbe de métriques $t \mapsto g_{t}=g+t k$.

Théorème 21. La variation infinitésimale de l'opérateur de Dirac en une métrique spinorielle $\gamma$ dans la direction d'un changement de métrique riemannienne $k$ est donnée par la formule

$$
\left(\frac{d}{d t} \gamma^{\mathscr{D}^{\gamma_{t}} \mid t=0}\right) \psi=-\frac{1}{2} \sum_{i=1}^{n} f_{i \cdot \gamma} D_{K_{g}\left(f_{\imath}\right)}^{\gamma} \psi+\frac{1}{4}\left(\delta^{g} k+d\left(\text { Trace }_{g} k\right)\right)_{\cdot \gamma} \psi,
$$

où $\left(f_{i}\right)$ est un repère g-orthonormé et où $\delta^{g}$ désigne l'opposé de la divergence sur les champs de 2-tenseurs symétriques.

Preuve. D'après le Théorème 20, nous avons

$$
\frac{d}{d t}\left({ }^{\gamma} \mathscr{D}^{\gamma_{t}}\right)_{\mid t=0}=-\frac{1}{2} \sum_{i=1}^{n} f_{i \cdot \gamma} D_{K_{g}\left(f_{i}\right)}^{\nu}+\sum_{i=1}^{n} f_{i \cdot \gamma}\left(D_{f_{i}}^{g} \frac{d}{d t}\left(G_{t}\right)_{g}^{-1 / 2}{ }_{\mid t=0}+\frac{d}{d t}{ }^{g} A_{f_{i} \mid t=0}^{g_{t}}\right) \text {. }
$$

Les 3-tenseurs qui apparaissent dans le second terme sont respectivement égaux à

$$
D^{g} \frac{d}{d t}\left(G_{t}\right)_{g}^{-1 / 2} \mid t=0(X, Y, Z)=-\frac{1}{2}\left(D_{X}^{g} k\right)(Y, Z)
$$

et

$$
\frac{d}{d t}{ }^{g} A_{\mid t=0}^{g_{t}}(X, Y, Z)=\frac{1}{2}\left(\left(D_{X}^{g} k\right)(Y, Z)+\left(D_{Y}^{g} k\right)(X, Z)-\left(D_{Z}^{g} k\right)(X, Y)\right)
$$

qui sont respectivement symétriques en les deux derniers et les deux premiers arguments. De ce fait, le terme en 3-forme dans le produit de Clifford donne une contribution nulle et le terme contracté est clairement celui qui apparaît dans le second terme de (21).

Remarque 23. L'espace $\mathscr{M} M$ des métriques riemanniennes sur $M$ a une géométrie intéressante; il possède en particulier une action du groupe $D M$ des difféomorphismes de $M$. Faire des variations d'une métrique riemannienne par action des difféomorphismes ne change pas la géométrie. Si nous voulons nous intéresser aux variations infinitésimales de la métrique qui changent la géométrie, il est donc utile de se restreindre aux variations qui sont transverses à cette action, ce qui est assuré en demandant que $k$ vérifie $\delta k=0$. (En effet son produit scalaire relatif à la 
métrique riemannienne standard de $\mathscr{M} M$ avec toute variation infinitésimale triviale $\mathscr{L}_{X} g$ est alors nul, l'opérateur de divergence $\delta$ étant à un facteur $\frac{1}{2}$ près l'adjoint de l'opérateur $X \mapsto \mathscr{L}_{X} g$.) Pour une telle variation la formule (21) se simplifie par disparition du terme en $\delta k$.

Nous nous intéressons maintenant aux variations des valeurs propres de l'opérateur de Dirac par changement de métrique. Nous supposons donc dans ce qui suit que nous nous plaçons en une métrique spinorielle $\gamma$ dont la partie métrique riemannienne subit une variation infinitésimale $k$ à laquelle nous associons la variation $g_{t}=g+t k$.

Rappelons que d'après un résultat de Rellich (cf. Theorem 3.9 de [13], voir aussi [13, Lemme 3.15]) la famille analytique d'opérateurs $\mathscr{D}^{\gamma_{t}}$ admet une décomposition spectrale $\left(\lambda_{t}^{i}, \Pi_{t}^{i}\right)$ analytique où, pour chaque $t, \lambda_{t}^{i}$ est une valeur propre et $\Pi_{t}^{i}$ le projecteur correspondant. Par une valeur propre fixée $\lambda$ de $\mathscr{D}^{\gamma}$, il passe $m$ branches $\lambda_{t}^{(1)}, \ldots, \lambda_{t}^{(m)}$ telles que $\lambda_{0}^{(1)}=\cdots=\lambda_{0}^{(m)}=\lambda$. Ainsi chaque variation infinitésimale $k$ de la métrique détermine les $m$ "dérivées premières" $d \lambda^{(r)} / d t_{\mid t=0}$ pour $1 \leqq r \leqq m(m=1$ lorsque la valeur propre est simple ou lorsque sa multiplicité est constante dans la direction $k$ ). Nous noterons $E_{\lambda}^{(r)}$ les sous-espaces propres.

Nous avons alors le résultat suivant

Théorème 24. Pour tout $r=1, \ldots, m$, la dérivée première de $\lambda$ correspondant à la branche $\lambda_{t}^{(r)}$ dans la direction $k$ s'écrit

$$
\left.\frac{d \lambda_{t}^{(r)}}{d t}\right|_{t=0}=-\frac{1}{2} \int_{M}\left(k, Q_{\psi^{(r)}}\right) v_{g},
$$

où $\psi^{(r)}$ est un élément unitaire (quelconque) du sous-espace $E_{\lambda}^{(r)}$ et où, pour tout champ de spineurs $\psi, Q_{\psi}$ est la forme bilinéaire symétrique réelle déterminée par

$$
Q_{\psi}(X, Y)=\frac{1}{2} \mathscr{R} e\left(\left(X{ }_{\cdot \gamma} D_{Y}^{\gamma} \psi, \psi\right)+\left(Y_{\cdot \gamma} D_{X}^{\gamma} \psi, \psi\right)\right) .
$$

Preuve. D'après le théorème de Rellich cité ci-dessus, $\psi^{(r)}$ se prolonge en une famille analytique $\psi_{t}^{(r)}$ de spineurs unitaires pour la norme $L^{2}$ propres pour $\mathscr{D}^{\gamma_{t}}$ relatif à la valeur propre $\lambda_{t}^{(r)}$.

Nous avons alors

$$
\left.\frac{d \lambda_{t}^{(r)}}{d t}\right|_{t=0}=\frac{d}{d t}\left(\int_{M}\left(\psi_{t}^{(r)},{ }^{\gamma} \mathscr{D}^{\gamma_{t}} \psi_{t}^{(r)}\right) v_{g}\right)_{\mid t=0}
$$

qui, après développement, donne trois termes. Les deux termes contenant la dérivée du vecteur propre donnent une contribution nulle car ils font intervenir le produit scalaire global de $\psi^{(r)}$ et de $d \psi^{(r)} / d t$ qui est nul à cause de la normalisation prise sur $\psi_{t}^{(r)}$. Il ne reste donc plus que le terme provenant de la dérivée de l'opérateur de Dirac. Nous pouvons donc utiliser la formule (21) ce qui donne la formule annoncée (24) en tenant compte du fait que le terme en $\delta_{g} k+d$ Trace $_{g} k$ donne lui-même une contribution nulle, car il agit de façon antisymétrique par produit de Clifford sur $\psi^{(r)}$.

Remarque 25. La forme quadratique $-\frac{1}{2} Q_{\psi^{(r)}}$ peut être considérée comme la $r^{\text {ième }}$ branche du gradient de $\lambda$ pour le produit scalaire standard sur $\mathscr{M} M$.

On peut aussi adopter un autre point de vue et considérer l'application $\psi \mapsto-\frac{1}{2} Q_{\psi}$ comme une forme quadratique définie sur le sous-espace propre $E_{\lambda}$ à valeurs dans l'espace des champs de formes bilinéaires sur $M$. Sa trace (par rapport 
à la métrique $L^{2}$ sur l'espace $E_{\lambda}$ ) s'interprète alors comme le gradient de la fonction $\eta \mapsto$ Trace $^{\gamma} \mathscr{D}_{\operatorname{lm} \Pi \Pi}^{\eta}$ où $\Pi$ désigne le projecteur spectral sur la somme des sous-espaces propres provenant de la déformation de $E_{\lambda}$ dans un voisinage de la métrique $\gamma$. Cette fonction n'est rien d'autre que la somme des valeurs propres issues de $\lambda$ par déformation.

La forme quadratique $Q_{\psi}$ associée à un spineur propre $\psi$ correspondant à une valeur propre non nulle $\lambda$ ne peut jamais être nulle car sa trace relative à g vaut $\lambda\|\psi\|^{2}$. Dans le cas d'une valeur propre non nulle, nous nous limiterons aux variations de métriques qui fixent le volume total, i.e. aux variations infinitésimales $k$ telles que $\int_{M}$ Trace $_{g} k v_{g}=0$. Nous dirons qu'une valeur propre non nulle $\lambda$ (éventuellement multiple) est critique si son gradient au sens défini dans la remarque 25 , i.e. la trace de la forme $Q$ prise sur $E_{\lambda}$, est un multiple de la métrique $g$ (nul automatiquement si $\lambda=0$ ).

Il est traditionnel d'appeler spineur harmonique les spineurs propres pour la valeur propre 0 . Dans le cas où la dimension $n$ de $M$ est paire, l'espace des spineurs harmoniques se décompose en la somme directe orthogonale de spineurs harmoniques de chiralités positive et négative. Comme la multiplication de Clifford par un vecteur échange les chiralités, il résulte immédiatement de $\left(24^{\prime}\right)$ que la restriction de $Q$ à chacun des deux sous-espaces est nulle. En particulier la trace de $Q$ est nulle sur l'espace des spineurs harmoniques, i.e. 0 est valeur propre critique pour toute métrique (pourvu qu'il y ait au moins un spineur harmonique non trivial).

Dans le cas où tous les spineurs harmoniques non triviaux ont même chiralité (ce qui ne peut se produire non trivialement que si $n$ est multiple de 4 à cause du théorème de.l'indice), la forme quadratique $Q$ est identiquement nulle sur l'espace des spineurs harmoniques. La dimension du noyau est alors minimale (encore par le théorème de l'indice); elle ne peut décroître dans un voisinage du point considéré dans l'espace des métriques.

Une question se pose naturellement: les métriques dont les spineurs harmoniques n'ont qu'une seule chiralité sont-elles génériques? A notre connaisance, elle est encore ouverte.

Une valeur propre de l'opérateur de Dirac ne change pas lorsqu'on varie la métrique par action des difféomorphismes (qui préservent la structure spinorielle). Il en résulte que la différentielle de la valeur propre dans l'espace $\mathscr{M} M$ des métriques riemanniennes s'annule sur l'espace tangent en $g$ à l'orbite des difféomorphismes ce qui implique que la divergence de la forme quadratique $Q_{\psi}$ doit être nulle pour tout champ de spineurs propres $\psi$ (cf. [4] Chap. 4). On peut vérifier ce fait directement par le calcul suivant (en omettant les indices $g$ et $\gamma$ ):

$$
\begin{aligned}
\left(\delta Q_{\psi}\right)(X) & =-\frac{1}{2} \sum_{i=1}^{n}\left(D_{f_{i}} Q_{\psi}\right)\left(f_{i}, X\right) \\
& =\frac{1}{2} \mathscr{R} e\left(\sum_{i=1}^{n}\left(\left(e_{i} \cdot D_{f_{i}, X}^{2} \psi, \psi\right)+\left(X \cdot D_{f_{i}} \psi, D_{f_{i}} \psi\right)\right)+\left(D_{Y} \psi, \mathscr{D} \psi\right)-(X \cdot \mathscr{D} \psi, \psi)\right) \\
& =\frac{1}{2} \mathscr{R} e\left(-\left(D_{X} \mathscr{D} \psi, \psi\right)+\left(D_{X} \psi, \mathscr{D} \psi\right)-(X \cdot \mathscr{D} \psi, \psi)\right),
\end{aligned}
$$

et la dernière expression est clairement nulle lorsque $\psi$ est un spineur propre. 
Examinons le cas particulier où le changement de métrique est un changement conforme. Si $h=e^{2 u} g$, alors la formule (20) se réduit à

$$
{ }^{\gamma} \mathscr{D}^{\eta} \psi=e^{-u}\left(\mathscr{D}^{\gamma} \psi+\frac{n-1}{2} d u_{\cdot \gamma} \psi\right) .
$$

ou encore

$$
{ }^{\gamma} \mathscr{D}^{\eta}=e^{((n+1) / 2) u} \circ \mathscr{D}^{\gamma} \circ e^{-((n-1) / 2) u} .
$$

Ce fait traduit l'existence d'un opérateur de Dirac conforme défini sur les sections du fibré des spineurs de poids conforme $-\frac{1}{2}(n-1)$, qui peuvent être l'un et l'autre définis purement en terme de la structure conforme (cf. [12] où il faut prendre garde que $T M$ est pris de poids conforme -1 , alors que nous prenons le fibré tangent de poids conforme +1 ).

La dérivée d'une valeur propre relativement à la variation $k=2 a g$ est égale d'après le Théorème 24 à

$$
\left.\frac{d \lambda_{t}^{(r)}}{d t}\right|_{t=0}=-\lambda \int_{M} a\left|\psi^{(r)}\right|^{2} v_{g} .
$$

La valeur propre 0 de l'opérateur de Dirac est toujours critique pour des variations conformes. C'est une conséquence de la formule $\left(26^{\prime}\right)$ qui fournit un isomorphisme entre les espaces de spineurs harmoniques pour deux métriques conformes.

Dans le cas de variations conformes, nous avons comme corollaire de la formule (26) le résultat suivant.

Proposition 27. Une valeur propre non nulle $\lambda$ est critique pour les variations conformes fixant le volume total, i.e. pour les variations infinitésimales $k=2$ ag telles que $\int_{M} a v_{g}=0$, si et seulement si la norme du spineur propre correspondant est constante.

Rappelons qu'un champ de $\gamma$-spineurs $\psi$ est appelé $\gamma$-spineur de Killing lorsqu'il satisfait la relation $D_{X}^{\gamma} \psi=-\frac{1}{n} \lambda X{ }_{\cdot \gamma} \psi$ pour tout vecteur tangent $X$ et un nombre réel $\lambda$. Un tel champ de spineurs est évidemment propre pour la valeur propre $\lambda$. On peut montrer que cette valeur propre est en valeur absolue la plus petite valeur propre de $\mathscr{D}$ et que tous les spineurs propres pour cette valeur propre sont des spineurs de Killing (cf. [10] et [11]). L'existence de spineurs de Killing impose des conditions très restrictives sur la métrique riemannienne $g$, en particulier $g$ est une métrique d'Einstein de courbure scalaire $4 \lambda^{2}(n-1) / n$. On a en outre comme conséquence immédiate des formules $(24)$ et $\left(24^{\prime}\right)$ la proposition suivante.

Proposition 28. S'il existe un spineur de Killing $\psi$ non trivial sur $(M, \gamma)$, la valeur propre $\lambda$ correspondante est critique en g parmi les métriques de volume total donné.

On observera que, puisque l'espace propre $E_{\lambda}$ est constitué de spineurs de Killing, la forme quadratique $Q$ (et pas seulement sa trace) est proportionnelle à $g$.

Donnons un exemple d'une variété spinorielle dont toutes les valeurs propres de l'opérateur de Dirac sont critiques.

Proposition 29. Les valeurs propres de l'opérateur de Dirac associé à la métrique spinorielle standard sur la sphère $S^{n}$ sont toutes critiques (pour les variations préservant le volume total). 
Preuve. Notons $\gamma$ la métrique spinorielle standard (de courbure sectionnelle constante et égale à 1). Les valeurs propres de $\mathscr{D}^{\gamma}$ sont égales en valeur absolue à $\pm(k+n / 2)$ pour $k \in \mathbf{N}$ (cf. [2]). Les spineurs propres correspondant aux valeurs propres minimales $\pm n / 2$ sont les spineurs de Killing. Le fibré en spineurs $\Sigma_{\gamma} S^{n}$ est trivialisé par une base orthonormée $\left(\psi_{\alpha}\right)$ de $E_{n / 2}$ (ou de $E_{-n / 2}$ ). Suivant [2], nous pouvons introduire les opérateurs $A^{+}=\left(\mathscr{D}+\frac{1}{2}\right)^{2}$ et $A^{-}=\left(\mathscr{D}-\frac{1}{2}\right)^{2}$ dont les espaces propres $\mathscr{A}_{k}^{ \pm}$sont les produits tensoriels $\mathscr{H}_{k} \otimes E_{ \pm(k+n / 2)}$ où $\mathscr{H}_{k}$ désigne l'espace des harmoniques sphériques d'ordre $k$, i.e. l'espace des fonctions propres du laplacien riemannien pour les valeurs propres $k(n+k-1)$.

On établit aisément les isomorphismes

$$
\mathscr{A}_{k}^{+}=E_{k+n / 2} \oplus E_{-(k-1+n / 2)}, \quad 1 \leqq k \leqq \infty,
$$

et

$$
\mathscr{A}_{k}^{-}=E_{-(k+n / 2)} \oplus E_{k-1+n / 2}, \quad 1 \leqq k \leqq \infty .
$$

Il découle directement de (24') que la trace de la forme quadratique $Q$ restreinte aux sous-espaces $\mathscr{A}_{k}^{ \pm}$est proportionnelle à $g$. Il suffit pour cela de l'évaluer sur une base d'éléments décomposés.

On termine la démonstration par un argument par récurrence utilisant les décompositions orthogonales $\left(30^{+}\right)$et $\left(30^{-}\right)$et le fait que la forme quadratique $Q$ est un multiple de la métrique $g$ pour tout spineur de Killing, i.e. pour tout élément de $\mathscr{A}_{0}^{ \pm}=E_{ \pm(n / 2)}$.

Remarque. Ce résultat est à rapprocher du résultat obtenu dans [3] pour le laplacien riemannien opérant sur les fonctions.

\section{Références}

1. Atiyah, M. F., Bott, R., Shapiro, A.: Clifford modules. Topology 3, [Suppl. 1] 3-38 (1964)

2. Bär, C.: Das Spektrum von Dirac-Operatoren. Dissertation, Univ. Bonn, 1990

3. Berger, M.: Sur les premières valeurs propres des variétés riemanniennes. Compositio Math. 26, 129-149 (1973)

4. Besse, A. L.: Einstein manifolds. Ergeb. Math. vol. 10. Berlin, Heidelberg, New York: Springer 1987

5. Binz, E., Pferschy, R.: The Dirac operator and the change of the metric. C.R. Math. Rep. Acad. Sci. Canada V 269-274 (1983)

6. Cartan, E.: La théorie des spineurs. Paris: Hermann 1937; and $2^{\text {éme }}$ édition, The theory of spinors. Paris: Hermann 1966

7. Cartan, E.: Notice sur les travaux scientifiques. Paris: Gauthier-Villars 1974

8. Chevalley, C.: Algebraic theory of spinors. New York: Columbia University Press 1954

9. Dabrowski, L., Percacci, R.: Spinors and diffeomorphisms. Commun. Math. Phys. 106, 691-704 (1986)

10. Friedrich, T.: Der erste Eigenwert des Dirac-Operators einer kompakten Riemannschen Mannigfaltigkeit nicht negativer Skalarkrümmung. Math. Nach. 97, 117-146 (1980)

11. Hijazi, O.: A conformal lower bound for the smallest eigenvalue of the Dirac operator and Killing spinors. Commun. Math. Phys. 104, 151-162 (1986)

12. Hitchin, N.: Harmonic spinors. Adv. Math. 14, 1-55 (1974)

13. Kato, T.: Perturbation theory for linear operators. Grundl. Math. Wiss. vol. 136. Berlin, Heidelberg, New York: Springer 1966

14. Kosmann, Y.: Dérivées de Lie des spineurs. Ann. Mat. Pura ed Appl. 91, 317-395 (1972)

15. Lawson, H. B., Michelsohn, M. L.: Spin geometry. Princeton Math. Series vol. 38, Princeton, NJ: Princeton University Press 1989 
16. Lichnerowicz, A.: Spineurs harmoniques. C.R. Acad. Sci. Paris A257, 7-9 (1963)

17. Milnor, J. W.: Remarks concerning Spin-manifolds. In: Differential and Combinatorial Topology. A symposium in honor of Morse, M., Cairns, S. S. (ed.), pp. 55-62. Princeton, NJ: Princeton University Press 1965

18. Ne'eman, Y.: Spinor-type fields with linear, affine and general coordinate transformations. Ann. Inst. Henri Poincaré XXVIII, 369-378 (1978)

19. Penrose, R., Rindler, R.: Spinors and space-time. Cambridge: Cambridge University Press 1984

20. Pferschy, R.: Die Abhängigkeit des Dirac-Operators von der Riemannschen Metrik. Dissertation Techn. Univ. Graz 1983

21. Weyl, H.: The classical groups, their invariants and representations. Princeton Math. Series 1, Princeton, NJ: Princeton University Press, Rev. ed. 1946; 8th edition, 1973

Communicated by A. Connes 
